\title{
p300-Catalyzed Lysine Crotonylation Promotes the Proliferation, Invasion, and Migration of HeLa Cells via Heterogeneous Nuclear Ribonucleoprotein A1
}

\author{
Xuesong Han, ${ }^{1}$ Xudong Xiang, ${ }^{2,3}$ Hongying Yang, ${ }^{2,3}$ Hongping Zhang, ${ }^{2,3}$ Shuang Liang, \\ Jie Wei, ${ }^{2,3}$ and Jing $\mathbf{Y u} \mathbb{1}^{2,3}$ \\ ${ }^{1}$ The First Affiliated Hospital of Kunming Medical University, Kunming, China \\ ${ }^{2}$ The Third Affiliated Hospital of Kunming Medical University, Kunming, China \\ ${ }^{3}$ Yunnan Cancer Center, Kunming, China \\ ${ }^{4}$ Pu'er People's Hospital, Pu'er, China
}

Correspondence should be addressed to Jing Yu; yu_jing_yn@163.com

Xuesong Han and Xudong Xiang contributed equally to this work.

Received 1 July 2020; Revised 13 October 2020; Accepted 28 November 2020; Published 7 December 2020

Academic Editor: Juan F. Santibanez

Copyright ( $\odot 2020$ Xuesong Han et al. This is an open access article distributed under the Creative Commons Attribution License, which permits unrestricted use, distribution, and reproduction in any medium, provided the original work is properly cited.

\begin{abstract}
Cervical carcinoma is the third most common cause of cancer in women with a significant challenge in clinical treatment. Human papillomavirus (HPV) is strongly responsible for cervical carcinoma. Here, we show the increased expression level of heterogeneous nuclear ribonucleoprotein A1 (HNRNPA1) in HPV-associated cervical cancer cells including HeLa, Caski, and SiHa cells, especially in HeLa cells. We provide the evidence that the expression of HNRNPA1 is closely related to HeLa cell proliferation, invasion, and migration. Emerging evidence show that histone modifications account for gene expression. Moreover, our results indicate that HNRNPA1 could be regulated by p300 through p300-mediated lysine crotonylation. Inhibition of p300 downregulated both the lysine crotonylation level and the HNRNPA1 expression. And p300-mediated lysine crotonylation participates in the regulation of HNRNPA1 on HeLa cell proliferation, invasion, and migration. Collectively, our study uncovers that p300-mediated lysine crotonylation enhances expression of HNRNPA1 to promote the proliferation, invasion, and migration of HeLa cells.
\end{abstract}

\section{Introduction}

Cervical carcinoma is the third most common cause of cancer in women worldwide [1]. Human papillomavirus (HPV) infection is supposed to be a major risk factor of cervical carcinoma [2], and about $95 \%$ of cases are caused by infections with high-risk HPV. However, there are limited therapeutic advances for HPV-associated cervical carcinoma. Thus, the prognosis marker of cervical cancers warrants further investigation.

As an oncogene, heterogeneous nuclear ribonucleoprotein A1 (HNRNPA1) was associated with cancer development and was supposed to be a promising therapeutic target in cancer. HNRNPA1 was involved in apoptosis of colon cancer cells [3]. In oral squamous cancer, HNRNPA1 could modulate the cell cycle and proliferation [4]. HNRNPA1 expression was related to the metastasis of breast cancer [5]. And HNRNPA1 activity affected the therapy of hepatocarcinoma [6]. Thus, the function of HNRNPA1 in $\mathrm{HPV}$-associated cervical carcinoma warranted further investigation. Here, we show that HNRNPA1 was upregulated in HPV-associated cervical cells. And inhibition of HNRNPA1 attenuated the proliferation, invasion, and migration of HeLa cells, which indicated that HNRNPA1 represented a potential target for HPV-associated cervical carcinoma.

Proteins such as histones and nonhistones are subject to a vast range of posttranslation modifications (PTMs) which mainly include methylation, phosphorylation, ubiquitination, 


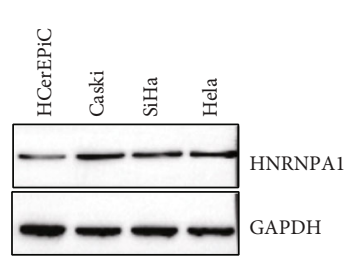

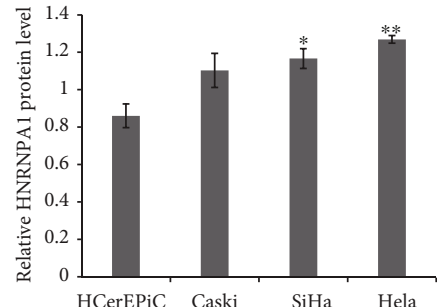

(a)

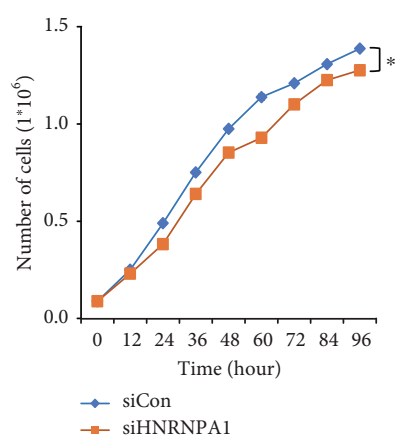

(c)
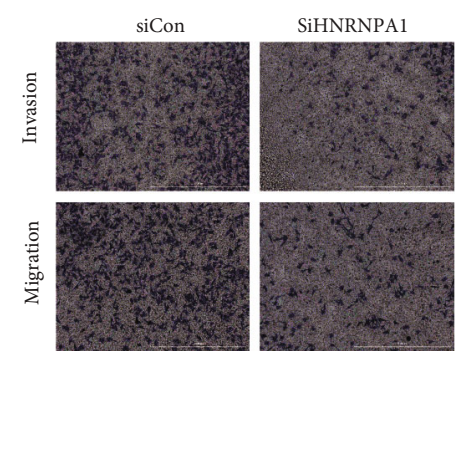

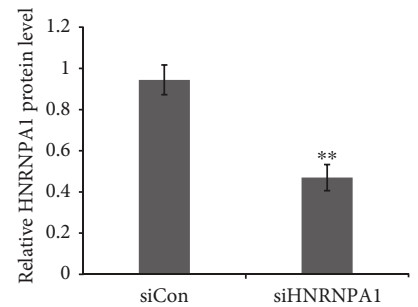

(b)

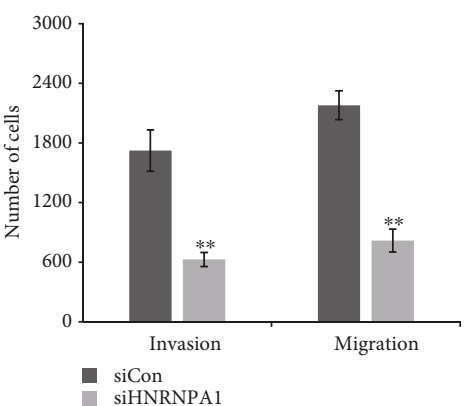

(d)

FIGURE 1: Knockdown of HNRNPA1 attenuated the proliferation, invasion, and migration of HeLa cells. (a) Western blot results showed the expression of HNRNPA1 in normal cervical epithelial cells (HCerEPiC) and HPV-associated cervical cancer cells (HeLa cells, Caski cells, and $\mathrm{SiHa}$ cells). The relative intensities of HNRNPA1/GAPDH were quantified using ImageJ. ${ }^{*} P<0.05$; ${ }^{* *} P<0.01$. (b) HNRNPA1 expression was measured by western blot in HeLa cells stably expressing nontargeting control (siCon) and siHNRNPA1. The relative intensities of HNRNPA1/GAPDH were quantified using ImageJ. ${ }^{* *} P<0.01$. (c) Effects of HNRNPA1 on HeLa cell proliferation were determined by CCK-8 kits. ${ }^{*} P<0.05$. (d) Transwell assay was performed to determine the invasion and migration of HeLa cells. The invasion and migration of HeLa cells were quantified using Image-Pro Plus 6.0. ${ }^{*} P<0.05$; ${ }^{* *} P<0.01$.

and acylation. The protein PTMs are identified to play roles in cancer progression such as tumorigenesis [7], oncogenic transformation [8], recurrence [9], and cancer therapy [10]. Moreover, protein PTMs were closely related to gene transcription. Besides acetylation, short-chain Lys acylations have been identified mainly including butyrylation (Kbu), propionylation (Kpr), and crotonylation (Kcr) [11]. More and more evidence demonstrated the role of $\mathrm{Kcr}$ in gene transcription $[12,13]$.

Different protein PTMs were carried out by different enzymes; these enzymes are frequently mutated or dysregulated in various types of cancer [14]. p300, as a transcriptional coactivator, contributed to cell proliferation [15], differentiation [16], apoptosis [17], and autophagy [18]. Except for histone acetyltransferase (HAT) activity of p300 [19], recent studies have illuminated that the histone crotonyltransferase activity of p300 and p300-mediated Kcr directly stimulates transcription [20]. A study has shown that p300 and p300-mediated Kcr participated in HNRNPA1 regulation [21]. However, the role of p300-mediated Kcr in HeLa cell proliferation, invasion, and migration which are regulated by HNRNPA1 remained obscure.

In present study, we investigated the effect of HNRNPA1 on proliferation, invasion, and migration of HeLa cells. Moreover, our results indicated that p300-mediated lysine crotonylation was able to regulate the expression of HNRNPA1 and in turn affect cell development.

\section{Materials and Methods}

2.1. Cell Culture. Human cervical cell lines (HeLa cells, Caski cells, and SiHa cells) and the normal cervical epithelial cell line HCerEPiC were purchased from ATCC (American Type Culture Collection, USA). Cells were maintained in Dulbecco's modified Eagle's medium (DMEM) supplemented with $10 \%$ fetal bovine serum (FBS) and $1 \%$ of penicillin solution. For sodium crotonate (NaCr, Alfa, B22235) treatment, cells were grown to $40 \%$ confluency and $20 \mathrm{mM}$ $\mathrm{NaCr}$ was added to the medium [22]. Cells were treated with the same concentration of $\mathrm{NaCl}$ in parallel as the control.

2.2. Cell Transfection. Stable knockdown of target genes was achieved by siRNA. The siRNA sequences used in this study were as follows: sip300 forward: AGAUACAAGCG AGGAAAACCA; reverse: GUUUUCCUCGCUUGUAUCU CC. siACSS2 forward: UUCUUAAAUAUCUAACUCCAA; reverse: GGAGUUAGAUAUUUAAGAAUC. siHNRNPA1 was purchased from Santa Cruz Biotechnology (Santa Cruz, CA). Cells were transfected with siRNA using Lipofectamine 3000 (Invitrogen, \# L3000-015) for $24 \mathrm{~h}$ according to the manufacturer's protocol. The knockdown efficiency was measured by western blots. For sodium crotonate treatment, transfected cells were grown to $40 \%$ confluency and $20 \mathrm{mM} \mathrm{NaCr}$ added to medium. 


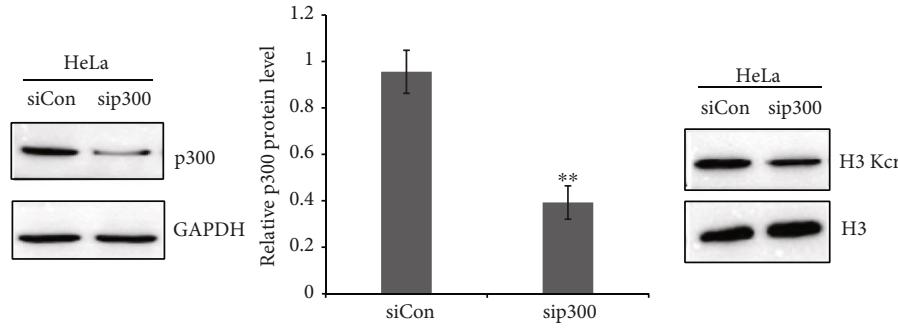

(a)

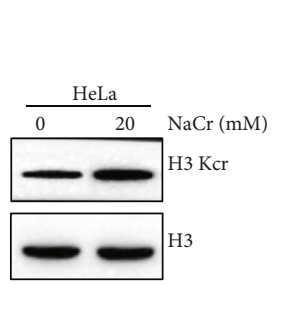

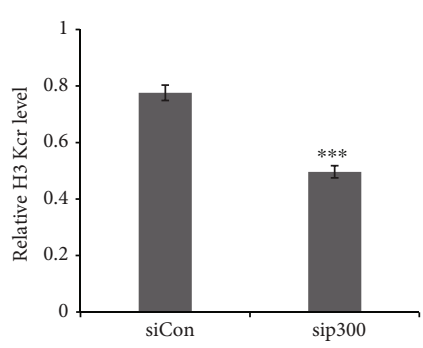

(b)

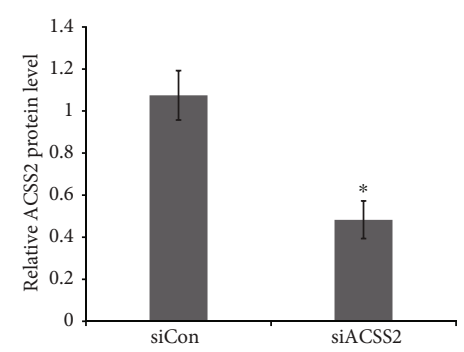

(d) (c)

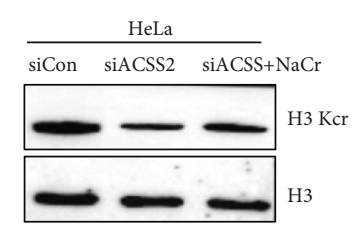

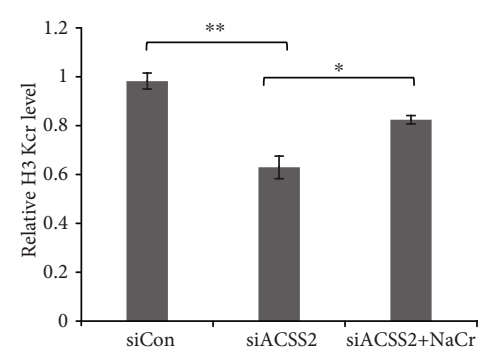

(e)

FIgURe 2: p300-mediated lysine crotonylation in HeLa cells. (a) p300 expression was measured by western blot in HeLa cells stably expressing nontargeting control (siCon) and sip300. The relative intensities of p300/GAPDH were quantified using ImageJ. ${ }^{* *} P<0.01$. (b) H3 Kcr expression was measured by western blot in HeLa cells stably expressing nontargeting control (siCon) and sip300. The relative intensities of $\mathrm{H} 3 \mathrm{Kcr} / \mathrm{H} 3$ were quantified using ImageJ. ${ }^{* * *} P<0.001$. (c) Effect of $\mathrm{NaCr}$ on $\mathrm{H} 3 \mathrm{Kcr}$ expression was determined by western blot. The relative intensities of $\mathrm{H} 3 \mathrm{Kcr} / \mathrm{H} 3$ were quantified using ImageJ. ${ }^{*} P<0.05$. (d) ACSS2 expression was measured by western blot in HeLa cells stably expressing nontargeting control (siCon) and siACSS2. The relative intensities of ACSS2/GAPDH were quantified using ImageJ. ${ }^{* *} P<0.01$. (e) $\mathrm{H} 3 \mathrm{Kcr}$ expression was determined by western blot in HeLa cell-treated siACSS2 or/and NaCr. The relative intensities of $\mathrm{H} 3 \mathrm{Kcr} / \mathrm{H} 3$ were quantified using ImageJ. ${ }^{*} P<0.05 ;{ }^{* *} P<0.01$.

2.3. Western Blots. Western blot analysis for a specific protein expression was performed. The protein was separated by sodium dodecyl sulfonate polyacrylamide gel electrophoresis (SDS-PAGE). The protein was transferred to a PVDF membrane by the wet transfer. After being blocked 1 hour in room temperature, the membrane was incubated by primary antibodies incorporating anti-histone H3 (ABclonal, A2348, 1:5000), anti-GAPDH (ABclonal, A19056, 1:5000), antipanKcr (PTM-501, 1:1000), anti-p300 (Abcam, ab59240, 1:2000), anti-ASCC2 (Abcam, ab234689, 1:2000), and anti-HNRNPA1 (Abcam, ab4791, $1: 1000$ ) at room temperature for 2 hours and then incubated with HRP-conjugated anti-rabbit (Abcam, 205717, 1:5000) and anti-mouse (Abcam, 205718, 1:5000) at room temperature for 1 hour.

\subsection{Cell Counting Kit (CCK-8) Assay. CCK-8 kit (Solarbio, \#} CA1210) was used to determine the cell proliferation. HeLa cells (5,000 cells/well) with or without HNRNPA1 knockdown and $\mathrm{NaCr}$ treatment were cultured. CCK- 8 solution was added, and cell numbers were then counted at 0,24 ,
48,72 , and $96 \mathrm{~h}$. Then, the optical density at $450 \mathrm{~nm}$ was measured by a microtiter plate reader.

2.5. Cell Migration and Invasion Assay. The cell migration and invasion assays were performed with Transwell chambers (Corning). For the migration assay, HeLa cells $\left(2 \times 10^{4}\right.$ in each well) with or without treatments were seeded in the upper Transwell chambers, and the lower chamber was filled with a medium containing 10\% FBS. For the invasion assay, the upper compartment was precoated with $100 \mu \mathrm{l}$ of Matrigel. After $24 \mathrm{~h}$ incubation, the cells in the upper surface were removed. Cells in the lower chamber were fixed with methanol and stained with Giemsa (Solarbio, \# G4640). The invasion and migration of HeLa cells were quantified using the Image-Pro Plus 6.0.

2.6. Statistical Analysis. All quantitative data are presented as the mean \pm SEM, and the differences were analyzed by twotailed Student's $t$ test or one-way ANOVA. One-way 


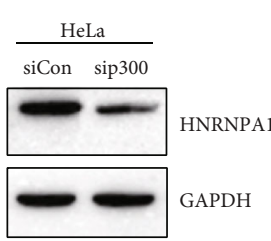

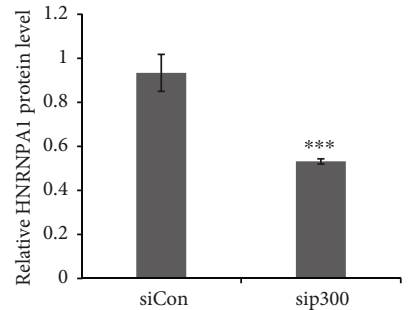

(a)
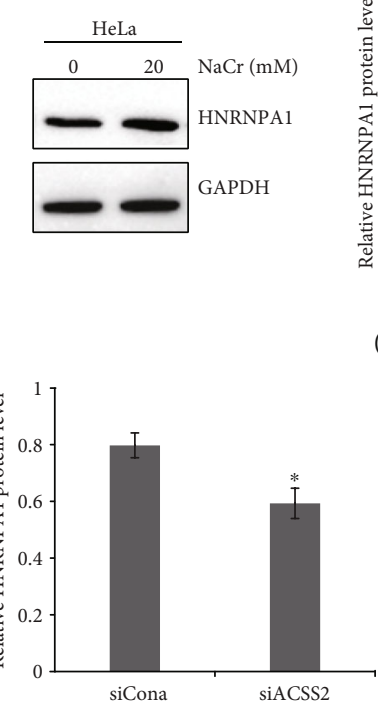

(c)

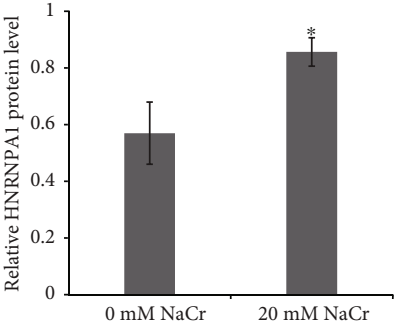

(b)

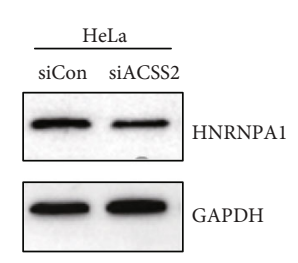

FIGURE 3: p300-mediated lysine crotonylation is responsible for HNRNPA1 expression in HeLa cells. (a) HNRNPA1 expression was measured by western blot in HeLa cells stably expressing nontargeting control (siCon) and sip300. The relative intensities of HNRNPA1/GAPDH were quantified using ImageJ. ${ }^{* *} P<0.001$. (b) HNRNPA1 expression was measured by western blot in NaCr-treated HeLa cells. The relative intensities of HNRNPA1/GAPDH were quantified using ImageJ. ${ }^{*} P<0.05$. (c) HNRNPA1 expression was measured by western blot in HeLa cells stably expressing nontargeting control (siCon) and siACSS2. The relative intensities of HNRNPA1/GAPDH were quantified using ImageJ. ${ }^{*} P<0.05$.

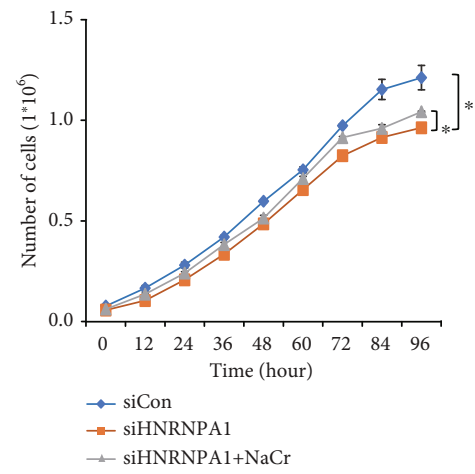

(a)
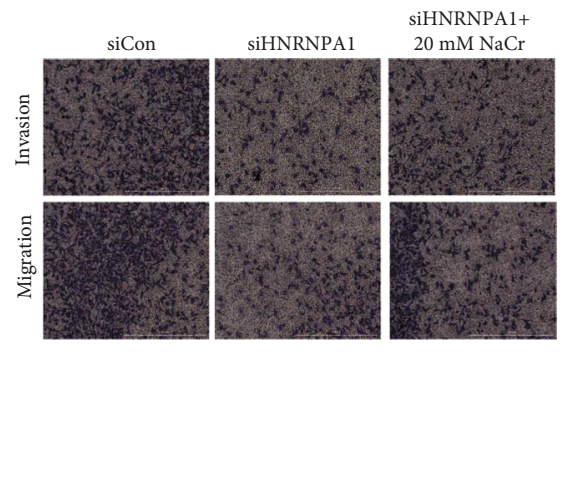

(b)

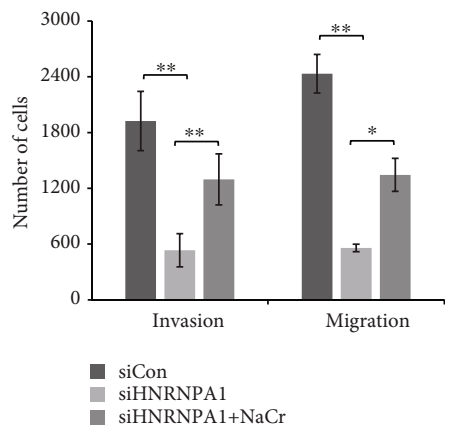

FIGURE 4: p300-mediated lysine crotonylation is involved in the regulation of HNRNPA1 on proliferation, invasion, and migration of HeLa cells. (a) Effects of HNRNPA1 or/and NaCr on HeLa cell proliferation were determined by CCK- 8 kit. ${ }^{*} P<0.05$. (b) Transwell assay was performed to determine the invasion and migration of HeLa cells treated with siHNRNPAl or/and NaCr. The invasion and migration of HeLa cells were quantified using Image-Pro Plus 6.0. ${ }^{*} P<0.05 ;{ }^{* *} P<0.01 ;{ }^{* * *} P<0.001$.

ANOVA with the Tukey post hoc test was used to analyze the differences among the different groups. Student's $t$ test was used for comparisons between two groups. Significance was accepted at $P<0.05$.

\section{Results and Discussion}

3.1. Knockdown of HNRNPA1 Attenuated the Proliferation, Invasion, and Migration of HeLa Cells. To identify the HNRNPA1 level in HPV-associated cervical cancer cells, we performed western blot assays to detect the protein level of HNRNPA1 in HeLa cells, Caski cells, SiHa cells, and HCerEPiC cells. As shown in Figure 1(a), the elevated level of HNRNPA1 could be observed in HPV-associated cervical cancer cells, particularly in HeLa cells. In order to determine the role of HNRNPA1 in HeLa cell development, HeLa cells expressing siRNA targeting HNRNPA1 were used (Figure 1(b)). As shown in Figure 1(c), inhibition of HNRNPA1 attenuated HeLa cell proliferation. In addition, HNRNPA1 silencing suppressed invasion and migration of HeLa cells (Figure 1(d)). Thus, our studies indicated that HNRNPA1 was involved in HeLa cell progression.

3.2. p300-Mediated Lysine Crotonylation in HeLa Cells. In order to determine whether p300 is responsible for Kcr in HeLa cells as reported, HeLa cells expressing siRNA targeting 
p300 were used. The knockdown efficiency of p300 was validated by western blot (Figure 2(a)). And p300 deficiency reduced the Kcr level in HeLa cells (Figure 2(b)). Based on the former result, p300-mediated Kcr could be induced by additional crotonyl-CoA, which was generated from $\mathrm{NaCr}$ [20]. When HeLa cells were treated with different concentrations of $\mathrm{NaCr}$, the increased level of $\mathrm{Kcr}$ could be observed (Figure 2(c)). Oppositely, knockdown acyl-CoA synthetase (ACSS2), which was known to produce acetyl-CoA, resulted in reduced Kcr level (Figures 2(d) and 2(e)). And $\mathrm{NaCr}$ administration in ACSS2-deficient HeLa cells could rescue the Kcr level (Figure 2(e)).

3.3. p300 and Lysine Crotonylation Is Responsible for HNRNPA1 Expression in HeLa Cells. Accumulating evidence indicated the effect of $\mathrm{Kcr}$ on gene transcription [23, 24]. It has been demonstrated that HNRNPA1 was a p300regulated lysine crotonylation protein [21]. In order to validate whether the expression of HNRNPA1 could be affected by $\mathrm{Kcr}$, we performed a western blot assay to detect the HNRNPA1 protein level under p300 knockdown. The expression level of HNRNPA1 was significantly decreased in HeLa cell transfected sip300 (Figure 3(a)). In addition, the expression level of HNRNPA1 could be upregulated by $\mathrm{NaCr}$ treatment (Figure 3(b)). And inhibition of ACSS2 led to decreased HNRNPA1 expression level (Figure 3(c)). In a word, our studies suggested that p300-catalyzed Kcr was involved in regulation of HNRNPA1 expression.

3.4. p300-Mediated Lysine Crotonylation Is Involved in the Regulation of HNRNPA1 on Proliferation, Invasion, and Migration of HeLa Cells. In order to determine the role of p300-catalyzed Kcr on the regulation of HNRNPA1 on HeLa cell progression, $\mathrm{NaCr}$ was used to treat $\mathrm{HeLa}$ cells with siHNRNPA1. As shown in Figure 4(a), $\mathrm{NaCr}$ administration rescued the inhibitory effect of HNRNPA1 knockdown on cell proliferation. Moreover, $\mathrm{NaCr}$ treatment also recovered the invasion and migratory abilities of HeLa cells with siHNRNPA1 (Figures 4(b) and 4(c)).

\section{Conclusions}

In the present work, we have demonstrated the function of HNRNPA1 in HPV-associated cervical cancer. The expression of HNRNPA1 was responsible for the development of HeLa cells. Knockdown of HNRNPA1 attenuated cell proliferation, invasion, and migration. We then detect the effect of p300 and p300-mediated Kcr on HNRNPA1 and Hele cell development. Inhibition of p300 dampened the $\mathrm{Kcr}$ level and HNRNPA1 expression. However, as we can see from Figure 2(b), knockdown of p300 could not completely abolish the histone $\mathrm{H} 3 \mathrm{Kcr}$ level, which indicated that the involvement of CBP on $\mathrm{Kcr}$. NaCr administration was able to induce Kcr and promote HNRNPA1 expression. On the contrary, the inhibition of crotonyl-CoA provider ACSS2 led to Kcr and HNRNPA1 reduction. Thus, our results indicated that p300-mediated Kcr was involved in HNRNPA1 expression. In order to identify the role of p300-mediated $\mathrm{Kcr}$ in HNRNPA1-regulated HeLa cell development, we performed proliferation assay and migration assay under $\mathrm{NaCr}$ administration. $\mathrm{NaCr}$ treatment attenuated the inhibitory effect of HNRNPA1 knockdown in HeLa cell proliferation, invasion, and migration.

Taken together, our investigation demonstrated that HNRNPA1 enhanced cell proliferation, invasion, and migration of HeLa cells through p300-mediated lysine crotonylation.

\section{Data Availability}

No data were used to support this study.

\section{Conflicts of Interest}

The authors declare that they have no competing interests.

\section{Acknowledgments}

The present study was funded by the Joint Project of the Yunnan Provincial Science and Technology Department-Kunming Medical University (grant no. 2017FE467-075).

\section{References}

[1] L. Shen, J. M. O'Shea, M. R. Kaadige et al., "Metabolic reprogramming in triple-negative breast cancer through Myc suppression of TXNIP," Proceedings of the National Academy of Sciences of the United States of America, vol. 112, pp. 54255430, 2015.

[2] M. Ngoma and P. Autier, "Cancer prevention: cervical cancer," ecancermedicalscience, vol. 13, p. 952, 2019.

[3] M. Fujiya, H. Konishi, M. K. M. Kamel et al., "MicroRNA-18a induces apoptosis in colon cancer cells via the autophagolysosomal degradation of oncogenic heterogeneous nuclear ribonucleoprotein A1," Oncogene, vol. 33, pp. 4847-4856, 2014.

[4] C. Yu, J. Guo, Y. Liu, J. Jia, R. Jia, and M. Fan, "Oral squamous cancer cell exploits hnRNP A1 to regulate cell cycle and proliferation," Journal of Cellular Physiology, vol. 230, pp. 22522261, 2015.

[5] T. J. Loh, H. Moon, S. Cho et al., "CD44 alternative splicing and hnRNP A1 expression are associated with the metastasis of breast cancer," Oncology Reports, vol. 34, pp. 1231-1238, 2015.

[6] S. Li, W. Wang, H. Ding et al., "Aptamer BC15 against heterogeneous nuclear ribonucleoprotein $\mathrm{A} 1$ has potential value in diagnosis and therapy of hepatocarcinoma," Nucleic Acid Therapeutics, vol. 22, pp. 391-398, 2012.

[7] Y. Xia, W. Yang, M. Fa et al., "RNF8 mediates histone H3 ubiquitylation and promotes glycolysis and tumorigenesis," Journal of Experimental Medicine, vol. 214, pp. 1843-1855, 2017.

[8] H. Aihara, T. Nakagawa, H. Mizusaki et al., "genic transformation via upregulation of cyclin D1," Molecular Cell, vol. 64, pp. 176-188, 2016.

[9] L. M. Katz, T. Hielscher, B. Liechty et al., "Loss of histone H3K27me3 identifies a subset of meningiomas with increased risk of recurrence," Acta Neuropathologica, vol. 135, pp. 955963, 2018.

[10] Q. Q. Li, J.-J. Hao, Z. Zhang et al., "Proteomic analysis of proteome and histone post-translational modifications in heat shock protein 90 inhibition-mediated bladder cancer therapeutics," Scientific Reports, vol. 7, article 201, 2017. 
[11] S. Zhao, X. Zhang, and H. Li, "Beyond histone acetylation-writing and erasing histone acylations," Current Opinion in Structural Biology, vol. 53, pp. 169-177, 2018.

[12] L. Kollenstart, A. J. L. de Groot, G. M. C. Janssen et al., "Gcn5 and Esal function as histone crotonyltransferases to regulate crotonylation-dependent transcription," Journal of Biological Chemistry, vol. 294, pp. 20122-20134, 2019.

[13] G. J. Gowans, J. B. Bridgers, J. Zhang et al., "Recognition of histone crotonylation by Taf14 links metabolic state to gene expression," Molecular cell, vol. 76, pp. 909-921.e3, 2019.

[14] R. Wang, M. Xin, Y. Li, P. Zhang, and M. Zhang, "The functions of histone modification enzymes in cancer," Current Protein \& Peptide Science, vol. 17, pp. 438-445, 2016.

[15] X. Hou, R. Gong, J. Zhan et al., "p300 promotes proliferation, migration, and invasion via inducing epithelial-mesenchymal transition in non-small cell lung cancer cells," BMC Cancer, vol. 18, p. 641, 2018.

[16] M. E. Rieger, B. Zhou, N. Solomon et al., "p300/ $\beta$-catenin interactions regulate adult progenitor cell differentiation downstream of WNT5a/protein kinase C (PKC)," Journal of Biological Chemistry, vol. 291, pp. 6569-6582, 2016.

[17] Z. Chen, W. Li, F. Qiu et al., “Aspirin cooperates with p300 to activate the acetylation of H3K9 and promote FasL-mediated apoptosis of cancer stem-like cells in colorectal cancer," Theranostics, vol. 8, pp. 4447-4461, 2018.

[18] S. Qian, Y. Han, Y. Shi et al., "Benzene induces haematotoxicity by promoting deacetylation and autophagy," Journal of Cellular and Molecular Medicine, vol. 23, pp. 1022-1033, 2019.

[19] X. Liu, L. Wang, K. Zhao et al., "The structural basis of protein acetylation by the p300/CBP transcriptional coactivator," Nature, vol. 451, pp. 846-850, 2008.

[20] B. R. Sabari, Z. Tang, H. Huang et al., "Intracellular crotonylCoA stimulates transcription through p300-catalyzed histone crotonylation,” Molecular Cell, vol. 58, pp. 203-215, 2015.

[21] H. Huang, D.-L. Wang, and Y. Zhao, "Quantitative crotonylome analysis expands the roles of p300 in the regulation of lysine crotonylation pathway," Proteomics, vol. 18, article e1700230, 2018.

[22] W. Wei, A. Mao, B. Tang et al., "Large-scale identification of protein crotonylation reveals its role in multiple cellular functions," Journal of Proteome Research, vol. 16, pp. 1743-1752, 2017.

[23] S. Liu, C. Xue, Y. Fang et al., "Global involvement of lysine crotonylation in protein modification and transcription regulation in rice," Molecular \& Cellular Proteomics, vol. 17, pp. 1922-1936, 2018.

[24] B. R. Sabari, Z. Tang, H. Huang et al., "Intracellular crotonylCoA stimulates transcription through p300-catalyzed histone crotonylation," Molecular Cell, vol. 69, p. 533, 2018. 